\title{
Kolmogorov laws for stratified turbulence
}

\author{
Pierre Augier $^{1} \dagger$, Sébastien Galtier ${ }^{2}$ and Paul Billant ${ }^{1}$ \\ ${ }^{1}$ LadHyX, CNRS, École Polytechnique, 91128 Palaiseau CEDEX, France \\ 2 Institut d'Astrophysique Spatiale, Université Paris-Sud, bâtiment 121, 91405 Orsay, France
}

(Received 9 February 2012; revised 22 May 2012; accepted 22 July 2012;

first published online 31 August 2012)

Following the Kolmogorov technique, an exact relation for a vector third-order moment $\boldsymbol{J}$ is derived for three-dimensional incompressible stably stratified turbulence under the Boussinesq approximation. In the limit of a small Brunt-Väisälä frequency, isotropy may be assumed which allows us to find a generalized 4/3-law. For strong stratification, we make the ansatz that $\boldsymbol{J}$ is directed along axisymmetric surfaces parameterized by a scaling law relating horizontal and vertical coordinates. An integration of the exact relation under this hypothesis leads to a generalized Kolmogorov law which depends on the intensity of anisotropy parameterized by a single coefficient. By using a scaling relation between large horizontal and vertical length scales we fix this coefficient and propose a unique law.

Key words: homogeneous turbulence, stratified flows, stratified turbulence

\section{Introduction}

The 4/5-law (Kolmogorov 1941) is one of the only exact results of the theory of three-dimensional homogeneous isotropic turbulence in the limit of large Reynolds numbers. Its more common expression is $\left\langle\left[\delta u_{L}(\boldsymbol{r})\right]^{3}\right\rangle=-(4 / 5) \varepsilon_{K} r$, where $\varepsilon_{K}$ is the mean kinetic energy dissipation rate per unit mass, $\delta u_{L}(\boldsymbol{r})=(\boldsymbol{u}(\boldsymbol{x}+\boldsymbol{r})-\boldsymbol{u}(\boldsymbol{x})) \cdot \boldsymbol{r} / r$ the longitudinal velocity increment and $\langle\cdot\rangle$ denotes a mean value. Alternatively, the Kolmogorov law can be expressed as $J_{L}(\boldsymbol{r})=-(4 / 3) \varepsilon_{K} r$, where $\boldsymbol{J}(\boldsymbol{r})=\left\langle|\delta \boldsymbol{u}(\boldsymbol{r})|^{2} \delta \boldsymbol{u}(\boldsymbol{r})\right\rangle$ is the vector third-order moment of the fluctuations; it is the so-called 4/3-law (Antonia et al. 1997). The Kolmogorov law is one of the most important results in the theory of homogeneous isotropic turbulence (Frisch 1995) for both fundamental and practical reasons. It gives the scaling law $\delta u \sim\left(\varepsilon_{K} r\right)^{1 / 3}$ and the exact coefficient of proportionality. The law expresses the nonlinear fluxes through scales in the inertial range as a function of measurable third-order moment quantities. Recent extensions of the isotropic Kolmogorov law have been made, to include quasi-geostrophic (Lindborg 2007), compressible (Galtier \& Banerjee 2011) or magnetized (Politano \& Pouquet 1998; Galtier 2008) turbulence.

The Kolmogorov 4/5-law comes from the theory of homogeneous isotropic turbulence where tensorial calculations are developed. Even if symmetries are used to simplify the analysis, it is difficult to follow the main steps of the derivation and the physical meaning can be hidden by technical difficulties. However, an easier alternative derivation exists (Monin \& Yaglom 1975; Podesta 2008) in which isotropy may be

$\dagger$ Present adress: Linné Flow Centre, KTH Mechanics, KTH, SE-100 44 Stockholm, Sweden. Email address for correspondence: augier@ladhyx.polytechnique.fr 
introduced only after an intermediate more general result, which is

$$
\nabla \cdot J=-4 \varepsilon_{K}
$$

It is important to emphasize that the assumption of isotropy must be dropped in many realistic situations such as geophysical turbulence, and more generally when rotation, stratification or an imposed magnetic field act on the flow. These physical ingredients drastically change the flow dynamics (with different invariants and instabilities) and break the isotropy. From a theoretical point of view, the lack of isotropy renders the derivation of the counterpart of the 4/3-law much more difficult. However, recent progress have been made in that direction for rotating or magnetized flows (Galtier 2009, 2011). Relaxing the isotropy assumption for the less restrictive hypothesis of axisymmetry, and adopting the critical balance viewpoint in which one makes the assumption of a scale-by-scale balance between the two time scales of the system (i.e. between the eddy turnover time and the wave time), the correlation space was assumed to become foliated in the sense that the vector third-order moments have a specific distribution. This new geometrical interpretation of the correlation space allows us to integrate (1.1) and find the counterpart of the 4/3-law for the axisymmetric turbulence studied.

In the present paper, we are mainly interested in purely stratified (non-magnetized, non-rotating) flows. It is well-known that geophysical turbulence in the Earth's fluid envelopes is strongly influenced by density stratification and rotation. Because the Brunt-Väisälä frequency $N$ characterizing the stratification is usually much larger than the local rotation frequency $f$, there are intermediate ranges of scales $(1-100 \mathrm{~km}$ in the atmosphere and $1 \mathrm{~m}-1 \mathrm{~km}$ in the oceans) where the Earth's rotation effect is negligible but where the stratification is strong (Riley \& Lelong 2000; Staquet \& Sommeria 2002).

The distinguishing attribute of these strongly stratified flows is a small horizontal Froude number $F_{h}=U /\left(N L_{h}\right)$, where $U$ is a characteristic velocity scale and $L_{h}$ an horizontal scale. Although the dynamics of strongly stratified turbulence has been a matter of debate for a long time, its understanding has significantly improved over the last decade and it is now well established that it is fully three-dimensional and strongly anisotropic. The characteristic vertical scale of a given structure is much smaller than its typical horizontal length scale and is of the order of the buoyancy length scale $L_{b}=U / N$ (Billant \& Chomaz 2001; Lindborg 2006). Most numerical simulations of strongly stratified turbulence have shown that the energy cascade is downscale, from large to small horizontal scales, as in isotropic homogeneous turbulence (Herring \& Metais 1989; Metais et al. 1996; Laval, McWilliams \& Dubrulle 2003; Riley \& de Bruyn Kops 2003; Waite \& Bartello 2004; Lindborg 2006; Brethouwer et al. 2007; Waite 2011; Kimura \& Herring 2012). Lindborg (2006) has shown that this strongly stratified direct energy cascade is associated with $k_{h}^{-5 / 3}$ energy spectra, where $k_{h}$ is the horizontal wavenumber. Such an inertial range exists however only if the buoyancy Reynolds number, $\mathscr{R}=R e F_{h}{ }^{2}$, where $R e$ is the usual Reynolds number, is large (Brethouwer et al. 2007). This condition requires very large Reynolds number since $F_{h} \ll 1$. This is largely fulfilled in geophysical flows but is much more difficult to achieve in direct numerical simulations or experiments. Nevertheless, such a strongly stratified inertial range has been observed in several numerical simulations and also in situ (Riley \& Lindborg 2008). However, direct non-local transfers toward horizontal scales of the order of the buoyancy length scale have been also found (Augier 2011; Waite 2011). Brethouwer et al. (2007) have shown that the strongly stratified inertial 
range should be followed at small scales by a nearly isotropic range since stratification effects weaken as the scale decreases down to the dissipative scale.

The purpose of the present paper is to derive a third-order structure function relation taking into account the anisotropy of strongly stratified turbulence in the stratified inertial range. Such a relation should be of interest for interpreting insitu measurements or numerical simulations. For example, Lindborg (1999), Cho \& Lindborg (2001) and Lindborg \& Cho (2001) performed statistical studies on aircraft measurements to compute the nonlinear fluxes using a 4/3-law derived for stratified rotating turbulence and for horizontal velocity increments. A vectorial anisotropic law applying especially to strongly stratified turbulence should allow one to gain further information from these data.

The paper is organized as follows. In $\S 2$ we derive an exact relation in terms of vector third-order moments and we discuss the weak stratification case for which a universal 4/3-law is proposed. Strong stratification is considered in $\S 3$ : we make the ansatz that the vector third-order moment is directed along axisymmetric surfaces and derive a generalized Kolmogorov law. Finally, our main results are summarized in $\S 4$.

\section{Exact relation for the vectorial third-order structure function}

\subsection{Governing equations}

The equations for an incompressible, non-diffusive and inviscid stably stratified fluid in the Boussinesq approximation are

$$
\begin{aligned}
\partial_{t} \boldsymbol{u}+\boldsymbol{u} \cdot \nabla \boldsymbol{u} & =-\nabla p+b \boldsymbol{e}_{z}, \\
\partial_{t} b+\boldsymbol{u} \cdot \nabla b & =-N^{2} u_{z}, \\
\boldsymbol{\nabla} \cdot \boldsymbol{u} & =0
\end{aligned}
$$

where $\boldsymbol{u}$ is the velocity, $u_{z}$ its vertical component, $p$ the rescaled pressure (energy per unit mass $), \quad N=\sqrt{-\left(g / \rho_{0}\right)(\mathrm{d} \bar{\rho} / \mathrm{d} z)}$ the constant Brunt-Väisälä frequency, $\boldsymbol{e}_{z}$ the vertical unit vector and $b=-\rho^{\prime} g / \rho_{0}$ the buoyancy perturbation, with $g$ the acceleration due to gravity, $\rho_{0}$ a reference density, $\bar{\rho}(z)$ the mean density and $\rho^{\prime}(\boldsymbol{x})$ the density perturbation. Note that it is assumed that $\rho_{0} \gg \bar{\rho}, \rho^{\prime}$ under the Boussinesq approximation.

Equations (2.1)-(2.2) have two invariants: the total energy and the potential enstrophy which are respectively (see e.g. Bartello 1995; Kurien, Smith \& Wingate 2006)

$$
E=\frac{1}{2}\left\langle|\boldsymbol{u}|^{2}\right\rangle+\frac{1}{2}\left\langle b^{2} / N^{2}\right\rangle, \quad Q=\frac{1}{2}\left\langle q^{2}\right\rangle,
$$

where $q=\left(g / \rho_{0}\right) \boldsymbol{\omega} \cdot \nabla \rho=-\boldsymbol{\omega} \cdot \nabla b-N^{2} \omega_{z}$ is the potential vorticity, with $\rho$ the total density and $\boldsymbol{\omega}=\boldsymbol{\nabla} \times \boldsymbol{u}$ the vorticity. The conservation of potential enstrophy does not imply an inverse cascade of energy like in two-dimensional turbulence or quasigeostrophic turbulence. Indeed, Lindborg (2006) has shown that the energy cascade is direct. In what follows, we shall focus on the first invariant, i.e. the energy.

\subsection{Stationary developed turbulence}

The following assumptions specific to fully developed turbulence will be made (Kolmogorov 1941; Frisch 1995). First, we assume the presence of a large-scale forcing $\mathscr{F}$ and a small-scale dissipation $\mathscr{D}$. Second, we take the long time limit for which a stationary state is reached with a finite mean total energy dissipation rate per unit mass. Third, we consider the infinite Reynolds number limit for which the mean total energy dissipation rate per unit mass tends to a finite limit, $\varepsilon$. 
It is simpler to consider the concept of fully developed stationary turbulence in spectral space in which the forcing and the dissipative terms are well localized. We use the definition of the Fourier transform adapted to a periodic three-dimensional box of side $\mathscr{L}$ :

$$
\hat{\boldsymbol{u}}(\boldsymbol{k}) \equiv \int_{\mathscr{L}^{3}} \boldsymbol{u}(\boldsymbol{x}) \mathrm{e}^{-\mathrm{i} \boldsymbol{k} \cdot \boldsymbol{x}} \frac{\mathrm{d}^{3} \boldsymbol{x}}{\mathscr{L}^{3}} .
$$

The equation of evolution of the energy of a mode $\boldsymbol{k}$ in Fourier space $\hat{E}(\boldsymbol{k})=$ $|\hat{\boldsymbol{u}}|^{2} / 2+\hat{b}^{2} /\left(2 N^{2}\right)$ can be written as

$$
\partial_{t} \hat{E}=\hat{T}+\hat{\mathscr{F}}-\hat{\mathscr{D}}
$$

where $\hat{T}$ is the conservative nonlinear transfer. The sums over all spectral modes of $\hat{\mathscr{F}}$ and $\hat{\mathscr{D}}$ are respectively the energy injection $P$ and the total dissipation $\varepsilon$, whereas the sum of $\hat{T}$ over all the spectral modes is null. For stationary turbulence, the time derivative is null and $P=\varepsilon$. In the limit of large Reynolds number, a wide separation of scales exists between large-scale forcing and small-scale dissipation. In between, we have an inertial range in which at first order the terms $\hat{\mathscr{F}}$ and $\hat{\mathscr{D}}$ are negligible. Turbulence is then characterized by a constant (non-zero) flux $\hat{\boldsymbol{\Pi}}$, with by definition $\partial \hat{\Pi}_{i} / \partial k_{i} \equiv-\hat{T}$. We now turn to the equivalent of (2.6) in the correlation space.

\subsection{Exact result for homogeneous stationary stratified turbulence}

In this subsection, we derive the evolution equation of two-point correlation functions which come from the inverse Fourier transform of $|\hat{\boldsymbol{u}}|^{2}$ and $|\hat{b}|^{2}$. Because our aim is to describe the inertial range we will neglect hereafter the forcing and dissipative terms. First, we start with the velocity correlation using the notation $\left\langle u_{i} u_{j}^{\prime}\right\rangle \equiv\left\langle u_{i}(\boldsymbol{x}) u_{j}\left(\boldsymbol{x}^{\prime}\right)\right\rangle$, where $\boldsymbol{x}^{\prime}=\boldsymbol{x}+\boldsymbol{r}$ and $\boldsymbol{r}$ is the separation vector. Using the homogeneity assumption and the divergence-free condition, we get the second-order correlation tensor equation

$$
\partial_{t}\left\langle u_{i} u_{j}^{\prime}\right\rangle=\partial_{r_{\ell}}\left[\left\langle u_{j}^{\prime} u_{\ell} u_{i}\right\rangle-\left\langle u_{i} u_{\ell}^{\prime} u_{j}^{\prime}\right\rangle\right]+\left\langle u_{i} b^{\prime} \delta_{j z}\right\rangle+\left\langle u_{j}^{\prime} b \delta_{i z}\right\rangle+\partial_{r_{i}}\left\langle p u_{j}^{\prime}\right\rangle-\partial_{r_{j}}\left\langle p^{\prime} u_{i}\right\rangle
$$

where Einstein's notation is used and $\delta_{i j}$ denotes the Kronecker tensor. The velocity-pressure correlation term is non-zero since isotropy has not been assumed up to now. When only the diagonal part of the energy tensor is retained their contribution disappears (Batchelor 1953) leading to

$$
\partial_{t}\left\langle u_{i} u_{i}^{\prime}\right\rangle=2 \nabla \cdot\left\langle\left(u_{i} u_{i}^{\prime}\right) \boldsymbol{u}\right\rangle+\left[\left\langle u_{z} b^{\prime}\right\rangle+\left\langle u_{z}^{\prime} b\right\rangle\right] .
$$

For the buoyancy correlation, the homogeneity hypothesis and the divergence freecondition give

$$
\partial_{t}\left\langle b b^{\prime}\right\rangle=2 \nabla \cdot\left\langle b b^{\prime} \boldsymbol{u}\right\rangle-N^{2}\left[\left\langle b u_{z}^{\prime}\right\rangle+\left\langle b^{\prime} u_{z}\right\rangle\right] .
$$

The combination of relations (2.8) and (2.9) gives eventually

$$
\partial_{t} E(\boldsymbol{r})=\nabla \cdot\left\langle\left[u_{i} u_{i}^{\prime}+b b^{\prime} / N^{2}\right] \boldsymbol{u}\right\rangle,
$$

where $E(\boldsymbol{r})=\left[\left\langle u_{i} u_{i}^{\prime}\right\rangle+\left\langle b b^{\prime}\right\rangle / N^{2}\right] / 2$. Equation (2.10) is nothing other than the inverse Fourier transform of (2.6) where the forcing and dissipative terms have been neglected; it can be written as $\partial_{t} E(\boldsymbol{r})=T(\boldsymbol{r})+\mathscr{F}(\boldsymbol{r})-\mathscr{D}(\boldsymbol{r})$. By identification, we see that

$$
T(\boldsymbol{r})=\nabla \cdot\left\langle\left[u_{i} u_{i}^{\prime}+b b^{\prime} / N^{2}\right] \boldsymbol{u}\right\rangle,
$$


which means that the nonlinear transfers in physical space can be expressed as a local divergence flux of correlation. This is the first intermediate result of the paper. It should be pointed out that $E(\boldsymbol{r})=T F^{-1}[\hat{E}]$ is a correlation function and should not be confused with the energy in physical space.

It is a classical result of homogeneous, isotropic, stationary, developed turbulence that $T(\boldsymbol{r})$ is equal in the inertial range to the opposite of $\Pi$, the energy flux from small to large wavenumbers, i.e. $T(\boldsymbol{r})=\mathscr{D}(\boldsymbol{r})-\mathscr{F}(\boldsymbol{r})=-\Pi=-\varepsilon$. As shown by Frisch (1995) or Lindborg (1999), this result remains valid for anisotropic homogeneous statistically stationary turbulence. Note that the relation $T(\boldsymbol{r})=-\varepsilon$ is also valid for an isotropic system without external forcing. However, for anisotropic non-stationary turbulence forced with isotropic structures, as in some grid turbulence experiments (Lamriben, Cortet \& Moisy 2011), the anisotropy develops with time from an isotropic flow. Hence, in this case, $T(\boldsymbol{r})$ is not constant and varies in the inertial region of the correlation space.

Since data analyses use generally structure functions, it is convenient to rewrite the relation $T(\boldsymbol{r})=-\varepsilon$ by introducing the vector third-order moment of increments:

$$
\nabla \cdot \boldsymbol{J}=\nabla \cdot\left\langle\left[|\delta \boldsymbol{u}|^{2}+(\delta b / N)^{2}\right] \delta \boldsymbol{u}\right\rangle=-4 \varepsilon .
$$

Equation (2.12) is an important intermediate result. It describes homogeneous stratified turbulence. This relation remains valid even when the turbulence is anisotropic. Note that the flux $\boldsymbol{J}$ can be divided into a kinetic flux and a potential flux which can be calculated independently. In practice, it is also interesting to quantify the term for exchange from kinetic to potential energies (Lumley 1964; Holloway 1988; Carnevale, Briscolini \& Orlandi 2001; Koshyk \& Hamilton 2001) which is $B(\boldsymbol{r})=\left[\left\langle\delta u_{z} \delta b\right\rangle / 2-\left\langle u_{z} b\right\rangle\right] / N^{2}$.

\subsection{Weak stratification limit}

When $N$ is small, the stratification effects may be seen as a correction to the dynamics of a non-stratified fluid. Then, we may expect that the assumption of isotropy still holds at first order. In this case, we can integrate expression (2.12) over a full sphere of radius $r$; after the application of the divergence theorem we obtain the universal law

$$
\left\langle\left[|\delta \boldsymbol{u}|^{2}+(\delta b / N)^{2}\right] \delta u_{L}\right\rangle=-\frac{4}{3} \varepsilon r,
$$

where $L$ denotes the longitudinal component of the vector, i.e. the one along the $\boldsymbol{r}$-direction. The stratification term appears as a correction to the 4/3-law derived in this form by Antonia et al. (1997). It is worth noting that a simple dimensional analysis performed on the universal law (2.13) leads to the isotropic spectral relation $E^{b}(k) \sim k^{-5 / 3}$ as for a passive scalar (Obukhov 1949).

\section{Kolmogorov law for anisotropic axisymmetric turbulence}

We now derive the counterpart of the Kolmogorov law for anisotropic axisymmetric turbulence starting from the general expression (2.12) which is exact for homogeneous turbulence. An application to strongly stratified turbulence will then be presented.

\subsection{Assumption on the direction of the flux $\mathbf{J}$}

The case of a strong stratification is more difficult to analyse since turbulence becomes anisotropic. However, we may assume that stratified turbulence remains axisymmetric, i.e. statistically invariant under rotation around the vertical axis. This implies that the flux $\boldsymbol{J}$ has a null azimuthal component and does not depend on the azimuthal 
(a)

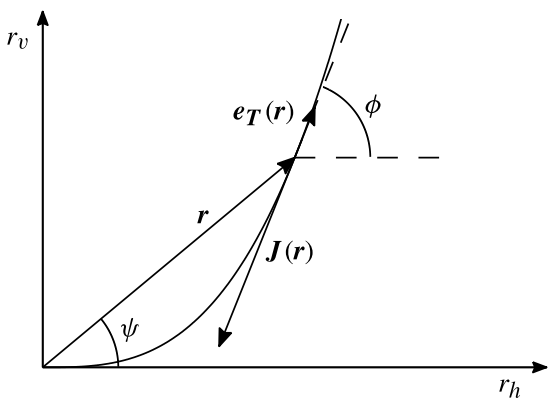

(b)

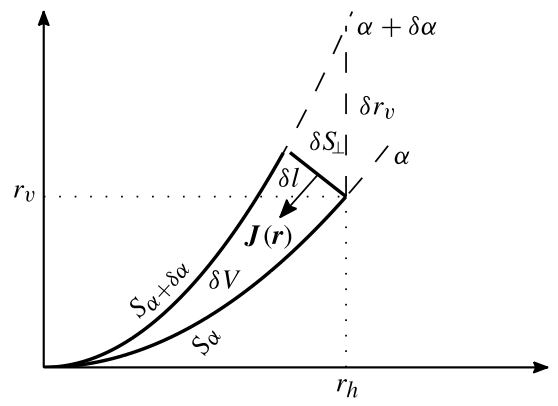

FIGURE 1. (a) Graphical representation of the assumption on the direction of the flux $\boldsymbol{J}$. (b) Infinitesimal volume $\delta V$ used for the integration. The infinitesimal surface closing the infinitesimal volume at the radius $r_{h}$ is given by $\delta S_{\perp}=2 \pi r_{h} \delta l$.

coordinate, i.e. $\boldsymbol{J}=J_{v}\left(r_{h}, r_{v}\right) \boldsymbol{e}_{v}+J_{h}\left(r_{h}, r_{v}\right) \boldsymbol{e}_{h}$, where $r_{v}$ is the vertical coordinate, $r_{h}=\sqrt{r_{x}^{2}+r_{y}^{2}}$ the radial coordinate and $\boldsymbol{e}_{\boldsymbol{v}}$ and $\boldsymbol{e}_{\boldsymbol{h}}$ the associated unit vectors. However, the axisymmetry alone is insufficient to integrate (2.12). For example, twodimensional and three-dimensional isotropic turbulence are both axisymmetric but lead to different Kolmogorov laws.

Hence, we have to specify the degree of anisotropy. Following Galtier (2009), we assume that the flux $\boldsymbol{J}$ is directed along axisymmetric surfaces parameterized by the power law

$$
r_{v}=f_{n}\left(r_{h}\right)=\alpha r_{h}{ }^{n},
$$

where $\alpha$ and $n$ are two real parameters. Because power laws are ubiquitous in turbulence this hypothesis seems natural. An interpretation of it will be given in $\S 3.4$. The variation of the coefficient $\alpha$ defines a continuous set of surfaces allowing the direction of $\boldsymbol{J}$ to be described in the entire correlation space. The exponent $n$ parametrizes the degree of anisotropy. For example, the two-dimensional isotropic case is obtained for $n=0$ with a horizontal flux $\boldsymbol{J}=J_{h} \boldsymbol{e}_{\boldsymbol{h}}$. The three-dimensional isotropic case is obtained for $n=1$ with a radial flux $\boldsymbol{J}=(\boldsymbol{r} \cdot \boldsymbol{J}) \boldsymbol{r} /|\boldsymbol{r}|^{2}$.

In the general case, the assumption that the flux $\boldsymbol{J}$ is directed along the curves (3.1) implies that $\boldsymbol{J}\left(r_{h}, r_{v}\right)=\boldsymbol{J}_{T}\left(r_{h}, r_{v}\right) \boldsymbol{e}_{T}\left(r_{h}, r_{v}\right)$, where $\boldsymbol{e}_{T}(\boldsymbol{r})$ is given by

$$
\boldsymbol{e}_{\boldsymbol{T}}\left(r_{h}, r_{v}\right)=\frac{\boldsymbol{e}_{\boldsymbol{h}}+f_{n}^{\prime}\left(r_{h}\right) \boldsymbol{e}_{\boldsymbol{v}}}{\sqrt{1+f_{n}^{\prime}\left(r_{h}\right)^{2}}}=\frac{\boldsymbol{e}_{\boldsymbol{h}}+n \tan \psi \boldsymbol{e}_{\boldsymbol{v}}}{\sqrt{1+n^{2} \tan ^{2} \psi}},
$$

with $\psi$ the angle between $\boldsymbol{r}$ and the horizontal plane (see figure $1 a$ ). The angle $\phi$ between $\boldsymbol{J}$ and the horizontal direction is therefore given by $\tan \phi=J_{v} / J_{h}=n \tan \psi$.

\subsection{Integration of (2.12)}

Having specified the direction of the flux $\boldsymbol{J}$, we can now integrate (2.12). We adopt a global method as is classically done in the isotropic case with an integration over a sphere in three dimensions and over a disk in two dimensions (Lindborg 1999). However, we have checked that the same result can be obtained through a local method (Augier 2011). In the anisotropic axisymmetric case, we have to consider an infinitesimal volume $\delta V$ drawn in figure $1(b)$ enclosed between two infinitesimally close surfaces $S_{\alpha}$ and $S_{\alpha+\delta \alpha}$ defined by (3.1). The volume is closed at the radius $r_{h}$ by an infinitesimal axisymmetric surface $\delta S_{\perp}=2 \pi \delta l$ perpendicular to the vector $\boldsymbol{J}$. 
Because the flux is assumed to flow over (and not through) the surfaces $S_{\alpha}$ and $S_{\alpha+\delta \alpha}$, the divergence theorem applied over the infinitesimal volume $\delta V$ yields

$$
\int_{\delta V} \nabla \cdot \boldsymbol{J} \mathrm{d}^{3} \boldsymbol{r}=\int_{S_{\alpha}, S_{\alpha+\delta \alpha}, S_{\perp}} \boldsymbol{J} \cdot \mathrm{d} \boldsymbol{S}=\delta S_{\perp} J_{T}\left(r_{h}, r_{v}\right) .
$$

Integrating (2.12) over the infinitesimal volume $\delta V$ leads therefore to

$$
J_{T}\left(r_{h}, r_{v}\right)=-4 \varepsilon \frac{\delta V\left(r_{h}, r_{v}, \delta \alpha\right)}{\delta S_{\perp}\left(r_{h}, r_{v}, \delta \alpha\right)} .
$$

In the limit $\delta \alpha \rightarrow 0$, the infinitesimal surface can be expressed as $\delta S_{\perp}\left(r_{h}, r_{v}, \delta \alpha\right)=$ $2 \pi r_{h} \cos \phi \delta r_{v}$ and the infinitesimal volume $\delta V$ can be computed as

$$
\delta V\left(r_{h}, r_{v}, \delta \alpha\right)=\int_{0}^{r_{h}} \mathrm{~d} r_{h}^{\prime} 2 \pi r_{h}^{\prime} \delta r_{v}^{\prime}=2 \pi \delta \alpha \int_{0}^{r_{h}} \mathrm{~d} r_{h}^{\prime} r_{h}^{\prime n+1}=2 \pi \delta r_{v} \frac{r_{h}{ }^{2}}{n+2} .
$$

This gives the following law: $J_{T}=-4 /(n+2) \varepsilon r_{h} / \cos \phi$ which can be rewritten as

$$
J_{T}\left(r_{h}, r_{v}\right)=-\frac{4}{n+2} \varepsilon r \cos \psi \sqrt{1+n^{2} \tan ^{2} \psi}=-\frac{4}{n+2} \varepsilon \sqrt{r_{h}^{2}+n^{2} r_{v}^{2}} .
$$

The flux is therefore given by

$$
\boldsymbol{J}\left(r_{h}, r_{v}\right)=-\frac{4 \varepsilon}{n+2}\left(r_{h} \boldsymbol{e}_{\boldsymbol{h}}+n r_{v} \boldsymbol{e}_{v}\right)
$$

It is the main result of the paper. We see that the vectorial law (3.7) for anisotropic axisymmetric turbulence has a form close to the isotropic case with, dimensionally, a linear dependence in $r$; however, anisotropy may be taken into account simply through the parameter $n$. Expression (3.7) is quite general and allows one to potentially describe different types of axisymmetric turbulence. In particular, the well-known three-dimensional isotropic law $J(r)=-(4 / 3) \varepsilon r$ is recovered for $n=1$ and the twodimensional law $J\left(r_{h}\right)=2 \varepsilon r_{h}$ is also recovered for $n=0$ (note that the sign depends on the type of cascade and it is fixed by hand).

\subsection{Anisotropy in strongly stratified turbulence}

We now discuss the main results known on the anisotropy of strongly stratified turbulent flows in order to find the appropriate value of the exponent $n$. As discussed in the Introduction, it is now well established that strongly stratified turbulence is strongly anisotropic and fully three-dimensional. Billant \& Chomaz (2001) have shown that the Boussinesq-Euler equations have an invariance in the limit of strong stratification (i.e. $\left.F_{h} \ll 1\right)$ that sets the characteristic vertical length scale $L_{v}$ to the buoyancy length scale $L_{b}=U / N$, where $U$ is a characteristic horizontal velocity. This scaling law is supported by simulations of stratified homogeneous turbulence (Riley \& Lelong 2000; Godeferd \& Staquet 2003; Waite \& Bartello 2004; Lindborg 2006; Brethouwer et al. 2007), three-dimensional stability analyses of various flows (Billant \& Chomaz 2000; Leblanc 2003; Otheguy, Chomaz \& Billant 2006) and experiments (Park, Whitehead \& Gnanadeskian 1994; Holford \& Linden 1999) provided that $\mathscr{R}=R e F_{h}^{2} \gg 1$, a condition ensuring negligible viscous effects.

Lindborg (2006) further assumed that this scaling law holds at each horizontal scale $l_{h}$ in strongly stratified turbulence, i.e. $l_{v} \sim \delta u_{h} / N$, where $\delta u_{h}$ is the characteristic 


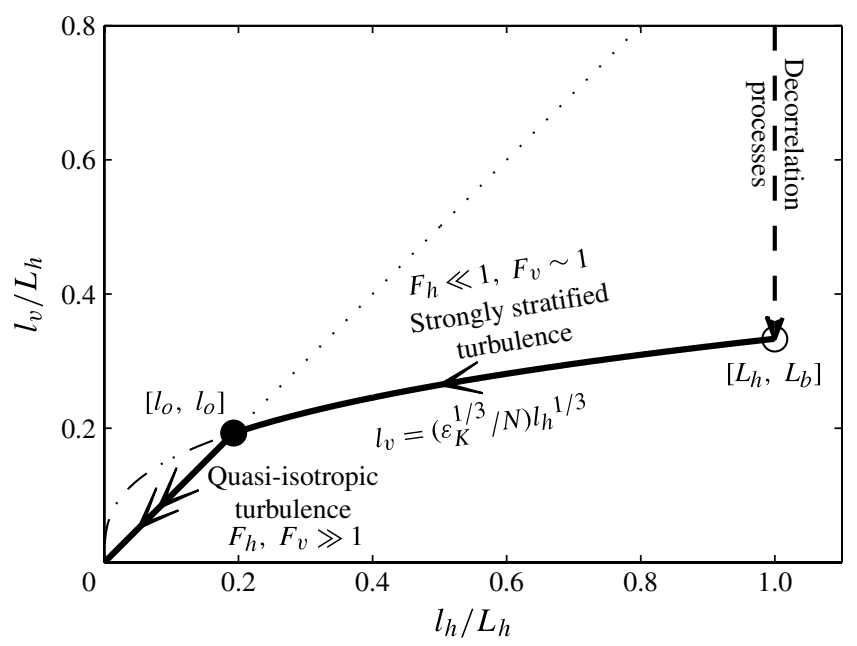

FIGURE 2. Schematic representation of the scaling laws relating vertical and horizontal characteristic length scales $l_{v}$ and $l_{h}$ in strongly stratified turbulence in real space. Arrows symbolize energy fluxes through different flow regimes. The bold line with the single arrow and the dashed-dotted line correspond to the scaling law (3.8). The bold line with the double arrow and the dotted line correspond to the scaling law $l_{v}=l_{h}$. The open and filled circles correspond to the large anisotropic structures and to the isotropic structures at the Ozmidov length scale, respectively.

horizontal velocity associated with $l_{h}$. This is equivalent to the so-called critical balance which states a scale-by-scale balance between the wave and the nonlinear time scales (Nazarenko \& Schekochihin 2011) and which is widely used for magnetized fluids (see e.g. Goldreich \& Sridhar 1995; Bigot, Galtier \& Politano 2008). Using this scaling law, Lindborg (2006) presented a theory and numerical evidence for a direct cascade of energy from large to small horizontal scales in strongly stratified turbulence. Through this cascade, the characteristic horizontal velocity $\delta u_{h}$ is given by $\delta u_{h}\left(l_{h}\right) \sim \varepsilon_{K}^{1 / 3} l_{h}^{1 / 3}$ as in homogeneous isotropic turbulence. As shown by many authors (Lindborg 2006; Brethouwer et al. 2007; Riley \& Lindborg 2008; Nazarenko $\&$ Schekochihin 2011), the combination of both previous scaling laws yields

$$
l_{v}=l_{o}\left(l_{h} / l_{o}\right)^{1 / 3}
$$

where $l_{o}=\left(\varepsilon_{K} / N^{3}\right)^{1 / 2}$ is the Ozmidov length scale (Lumley 1964; Ozmidov 1965). This relation between horizontal and vertical characteristic length scales shows that structures have a pancake shape with $l_{v} \ll l_{h}$ when $l_{h} \gg l_{o}$. However, the isotropy is recovered when $l_{h}$ is of the order of the Ozmidov length scale $l_{o}$. For scales $l_{h}$ smaller than $l_{o}$, the horizontal Froude number $F_{h}\left(l_{h}\right)=u_{h}\left(l_{h}\right) /\left(N l_{h}\right)=\left(l_{o} / l_{h}\right)^{2 / 3}$ is larger than unity so that they are only weakly affected by the stratification. This led Brethouwer et al. (2007) to argue that the strongly stratified inertial range exists only up to the Ozmidov length scale and that there is a transition to quasi-isotropic homogeneous turbulence for scales smaller than the Ozmidov length scale and down to the Kolmogorov length scale.

These two regimes are sketched in figure 2 in the space $\left(l_{h}, l_{v}\right)$. The turbulence is assumed to be forced at the large horizontal scale $L_{h}$ with a vertically invariant 
forcing (i.e. such as $L_{v} \gg L_{b}$ ) as in the numerical simulations of Waite \& Bartello (2004), Lindborg (2006) and Brethouwer et al. (2007) and in the experiments of Praud, Fincham \& Sommeria (2005). Large pancake structures (represented by an open circle in figure 2) arise spontaneously through decorrelation processes which are ubiquitous in stratified flows. Indeed, different mechanisms leading to a vertical decorrelation have been described in different contexts, such as the zigzag instability (Billant \& Chomaz 2000), internal wave resonances (Leblanc 2003), weakly nonlinear wave turbulence (Caillol \& Zeitlin 2000) and the Lilly decorrelation process (Lilly 1983). The two first processes select a vertical scale which is $L_{b}$, i.e. the buoyancy length scale.

Although the scaling law (3.8) has a power-law dependence as (3.1), their physical meanings are completely different. Equation (3.8) relates the horizontal and vertical characteristic length scales of structures whereas (3.1) specifies the direction of the flux $\boldsymbol{J}$ in the correlation space $\left(r_{h}, r_{v}\right)$.

The relation (3.8) means that the typical correlation length in the vertical direction $l_{v}$ is smaller than the one in the horizontal direction $l_{h}$. Therefore, if we assume that the isolines of correlation are ellipsoidal in the correlation space $r_{h}, r_{v}$, they should be given by $\left(r_{h} / l_{h}\right)^{2}+\left(r_{v} / l_{v}\right)^{2}=1$. In other words, the isocorrelation lines are flattened in the vertical direction. By using (3.8), the ellipse equation can be rewritten as $r_{h}{ }^{2}+\left(l_{h} / l_{o}\right)^{4 / 3} r_{v}{ }^{2}=l_{h}^{2}$. The aspect ratio of the elliptic contours of constant correlation, $p=l_{h} / l_{v}=\left(l_{h} / l_{o}\right)^{2 / 3}$, is therefore not constant but varies with $l_{h}$. It decreases from values larger than unity for the strongly stratified turbulent cascade $\left(l_{h} \gg l_{o}\right)$ down to unity at the Ozmidov length scale where the isotropy is recovered. We can note that the isolines of the flux modulus $|\boldsymbol{J}|$ are also ellipsoidal according to (3.6) and such that $r_{h}{ }^{2}+n^{2} r_{v}{ }^{2}=$ const., but the aspect ratio $n$ is constant. Furthermore, the isocorrelation lines and the isoflux lines do not necessarily have the same shape so that it seems not possible to deduce $n$ from these considerations.

\subsection{Alternative derivation of (3.7)}

In fact, a result equivalent to (3.7) can be derived without using the hypothesis (3.1) and this will give some indications of how the parameter $n$ can be deduced. Indeed, another way to solve the divergence equation (2.12) is to use the Helmholtz decomposition to write the flux $\boldsymbol{J}=\nabla G+\boldsymbol{\nabla} \times \boldsymbol{H}$. Since stratified turbulence is statistically invariant under inversion of the vertical axis, the pseudo-vector $\boldsymbol{\nabla} \times \boldsymbol{J}$ should be equal to zero implying $\boldsymbol{H}=0$. Note that this symmetry argument does not apply to rotating and magnetized turbulence. In any case, the potential $G$ satisfies a Poisson equation $\nabla^{2} G=-4 \varepsilon$. This equation has to be solved in a closed domain bounded by a surface $\Sigma$ corresponding to the largest scales $L_{h}$ and $L_{v}$. Assuming that the solution is non-singular at $r_{h}=r_{v}=0$, the general solution can be written $G=G_{1}+G_{2}$ where $G_{2}$ satisfies $\nabla^{2} G_{2}=0$ and

$$
G_{1}=-\frac{2 \varepsilon}{2+P_{G}^{2}}\left(r_{h}^{2}+P_{G}^{2} r_{v}^{2}\right)
$$

with $P_{G}$ a constant. The contours of constant $G_{1}$ are elliptic with an aspect ratio equal to $P_{G}$.

If $\Sigma$ is an oblate spheroid defined by $\left(r_{h} / L_{h}\right)^{2}+\left(r_{v} / L_{v}\right)^{2}=1$ the complementary solution $G_{2}$ can be found by separation of variables in terms of oblate spheroidal 
harmonics (Abramowitz \& Stegun 1965). In any case, it will depend on the particular boundary conditions (i.e. forcing) imposed at the largest scales, i.e. on $\Sigma$.

It is then straightforward to compute the divergent part of the flux

$$
\boldsymbol{J}=-\frac{4 \varepsilon}{2+P_{G}{ }^{2}}\left(r_{h} \boldsymbol{e}_{h}+P_{G}^{2} r_{v} \boldsymbol{e}_{v}\right)+\nabla G_{2} .
$$

Remarkably, the first part of (3.10) has exactly the same form as (3.7) and implies directly that this component of the flux is directed along lines defined as in the power law (3.1) with a constant $n=P_{G}{ }^{2}$. In addition, if the flux imposed by the forcing at the largest scales satisfies (3.1), i.e. $\nabla G_{2}=0$ on $\Sigma$, then we have $G_{2}=0$ for any $r_{h}$ and $r_{v}$. In this case, the constant $n$ is fixed by the direction of the flux at the largest scales. In other words, the structure of the flux $\boldsymbol{J}$ at all scales is entirely determined by the flux forced at the largest scales.

This suggests that the parameter $n$ may depend on the type of forcing for each particular experiment or simulation. Nevertheless, we can assume that the largest scales are in a balanced state, i.e. that they fulfil the scaling laws $L_{v}=L_{b}$ and $\delta u_{v} / \delta u_{h} \sim F_{h}=L_{b} / L_{h}$ (Billant \& Chomaz 2001). Then, we can assume $J_{v} / J_{h} \sim$ $\delta u_{v} / \delta u_{h}$ at the energy-containing scales $r_{h} \simeq L_{h}$ and $r_{v} \simeq L_{v}$ to obtain $n=P_{G}{ }^{2} \sim 1$. This scaling analysis suggests that the value of $n$ should be close to the isotropic value even for a strongly anisotropic flow. It would be interesting to compute the flux $\boldsymbol{J}$ in experiments or numerical simulations to test our predictions and to determine whether or not strongly stratified turbulence is associated to a universal value of $n$.

\section{Conclusion}

In this paper an exact relation for homogeneous, incompressible, stably stratified turbulence has been derived. This relation shows the modifications brought to the Kolmogorov vectorial relation by the stratification. When the Brunt-Väisälä frequency is small, isotropy is still expected at first order and an universal 4/3-law may be derived.

For strong stratification anisotropy can no longer be neglected. The ansatz is made that the flux of correlation $\boldsymbol{J}$ flows along axisymmetric surfaces whose form depends on the intensity of anisotropy. Under this hypothesis the exact relation has been integrated and a generalized Kolmogorov law has been found. An alternative derivation of this generalized law based on the Helmholtz decomposition of the flux has also been presented. It shows that the intensity of anisotropy of the flux should depend on the large scales of the flow, i.e. on the type of forcing. An explicit prediction for the flux $\boldsymbol{J}$ has been obtained assuming that the large vertical scales fulfil the scaling law $L_{v} \simeq L_{b}$.

It would be interesting to check these predictions and in particular the ones about the direction of the flux $\boldsymbol{J}$ and its dependence on the large-scale structures. In the case of rotating turbulence, Lamriben et al. (2011) have recently measured experimentally anisotropic energy transfers. However, in these experiments the forcing is isotropic and anisotropy develops with time so that the nonlinear transfer $T(\boldsymbol{r})$ (see (2.11)) is not constant. For this reason, it might be easier to test our predictions for the flux $\boldsymbol{J}$ for statistically stationary turbulence by means of numerical simulations of forced strongly stratified turbulence. 


\section{Acknowledgements}

The authors wish to thank F. Moisy and E. Lindborg for interesting discussions and anonymous reviewers for helpful comments. Financial support from Institut universitaire de France is gratefully acknowledged. This work was supported by the National Research Agency (ANR-06-BLAN-0363).

\section{REFERENCES}

Abramowitz, M. \& Stegun, I. A. 1965 Handbook of Mathematical Functions. Dover.

Antonia, R. A., Ould-Rouis, M., Anselmet, F. \& Zhu, Y. 1997 Analogy between predictions of Kolmogorov and Yaglom. J. Fluid Mech. 332, 395-409.

AugIer, P. 2011 Turbulence in strongly stratified fluids: cascade processes. PhD thesis, LadHyX, Ecole Polytechnique, http://tel.archives-ouvertes.fr/tel-00697245.

BARTELlO, P. 1995 Geostrophic adjustment and inverse cascades in rotating stratified turbulence. J. Atmos. Sci. 52 (24), 4410-4428.

BAtCheloR, G. K. 1953 The Theory of Homogeneous Turbulence. Cambridge University Press.

Bigot, B., Galtier, S. \& Politano, H. 2008 Energy decay laws in strongly anisotropic MHD turbulence. Phys. Rev. Lett. 100, 074502-4.

Billant, P. \& Chomaz, J.-M. 2000 Three-dimensional stability of a vertical columnar vortex pair in a stratified fluid. J. Fluid Mech. 419, 65-91.

Billant, P. \& Chomaz, J.-M. 2001 Self-similarity of strongly stratified inviscid flows. Phys. Fluids 13, 1645-1651.

Brethouwer, G., Billant, P., Lindborg, E. \& Chomaz, J.-M. 2007 Scaling analysis and simulation of strongly stratified turbulent flows. J. Fluid Mech. 585, 343-368.

Caillol, P. \& Zeitlin, V. 2000 Kinetic equations and stationary energy spectra of weakly nonlinear internal gravity waves. Dyn. Atmos. Oceans 32, 81-112.

Carnevale, G. F., Briscolini, M. \& Orlandi, P. 2001 Buoyancy- to inertial-range transition in forced stratified turbulence. J. Fluid Mech. 427, 205-239.

Cho, J. Y. N. \& Lindborg, E. 2001 Horizontal velocity structure functions in the upper troposphere and lower stratosphere 1. Observations. J. Geophys. Res. 106 (D10), 10223-10232.

FRISCH, U. 1995 Turbulence: The Legacy of A.N. Kolmogorov. Cambridge University Press.

Galtier, S. 2008 von Karman-Howarth equations for Hall magnetohydrodynamic flows. Phys. Rev. E 77, 015302(R)-4.

Galtier, S. 2009 Exact vectorial law for homogeneous rotating turbulence. Phys. Rev. E 80, 046301-9.

Galtier, S. 2011 Third-order Elsässer moments in axisymmetric MHD turbulence. C. R. Phys. 12, 151-159.

GAltier, S. \& BANERJEe, S. 2011 Exact relation for correlation functions in compressible isothermal turbulence. Phys. Rev. Lett. 107, 134501-4.

Godeferd, F. S. \& Staquet, C. 2003 Statistical modelling and direct numerical simulations of decaying stably stratified turbulence. Part 2. Large-scale and small-scale anisotropy. J. Fluid Mech. 486, 115-159.

Goldreich, P. \& SRIDHAR, S. 1995 Towards a theory of interstellar turbulence II. Strong Alfvénic turbulence. Astrophys. J. 438, 763-775.

Herring, J. R. \& Metais, O. 1989 Numerical experiments in forced stably stratified turbulence. J. Fluid Mech. 202, 97-115.

Holford, J. M. \& Linden, P. F. 1999 Turbulent mixing in a stratified fluid. Dyn. Atmos. Oceans 30 (2-4), 173-198.

Holloway, G. 1988 The buoyancy flux from internal gravity wave breaking. Dyn. Atmos. Oceans 12, $107-125$.

Kimura, Y. \& Herring, J. R. 2012 Energy spectra of stably stratified turbulence. J. Fluid Mech. 698, 19-50.

Kolmogorov, A. N. 1941 Dissipation of energy in the locally isotropic turbulence. Dokl. Akad. Nauk SSSR 32, 16-18. 
Koshy,, J. N. \& Hamilton, K. 2001 The horizontal kinetic energy spectrum and spectral budget simulated by a high-resolution troposphere-stratosphere-mesosphere GCM. J. Atmos. Sci. 58 (4), 329-348.

Kurien, S., Smith, L. \& Wingate, B. 2006 On the two-point correlation of potential vorticity in rotating and stratified turbulence. J. Fluid Mech. 555, 131-140.

Lamriben, C., Cortet, P.-P. \& Moisy, F. 2011 Direct measurements of anisotropic energy transfers in a rotating turbulence experiment. Phys. Rev. Lett. 107 (2), 024503.

Laval, J. P., McWilliams, J. C. \& Dubrulle, B. 2003 Forced stratified turbulence: successive transitions with Reynolds number. Phys. Rev. E 68 (3), 036308.

Leblanc, S. 2003 Internal wave resonances in strain flows. J. Fluid Mech. 477, 259-283.

LILLY, D. K. 1983 Stratified turbulence and the mesoscale variability of the atmosphere. J. Atmos. Sci. 40, 749-761.

LINDBORG, E. 1999 Can the atmospheric kinetic energy spectrum be explained by two-dimensional turbulence? J. Fluid Mech. 388, 259-288.

LindBorg, E. 2006 The energy cascade in a strongly stratified fluid. J. Fluid Mech. 550, 207-242.

LINDBORG, E. 2007 Third-order structure function relations for quasi-geostrophic turbulence. J. Fluid Mech. 572, 255-260.

LindBorg, E. \& CHO, J. Y. N. 2001 Horizontal velocity structure functions in the upper troposphere and lower stratosphere 2. Theoretical considerations. J. Geophys. Res. 106 (D10), 10233-10241.

Lumley, J. L. 1964 The spectrum of nearly inertial turbulence in a stably stratified fluid. J. Atmos. Sci. 21 (1), 99-102.

Metais, O., Bartello, P., Garnier, E., Riley, J. J. \& Lesieur, M. 1996 Inverse cascade in stably stratified rotating turbulence. Dyn. Atmos. Oceans 23 (1-4), 193-203.

Monin, A. S. \& Yaglom, A. M. 1975 Statistical Fluid Mechanics, vol. 2. MIT.

NAZARenko, S. V. \& SCheKochinin, A. A. 2011 Critical balance in MHD, rotating and stratified turbulence: towards a universal scaling conjecture. J. Fluid Mech. 677, 134-153.

Oвuкhov, А. M. 1949 Structure of the temperature field in turbulent flows. Izv. Akad. Nauk. SSSR, Geogr. Geofiz. 13 (58).

Otheguy, P., Chomaz, J.-M. \& Billant, P. 2006 Elliptic and zigzag instabilities on co-rotating vertical vortices in a stratified fluid. J. Fluid Mech. 553, 253-272.

OzMIDov, R. V. 1965 On the turbulent exchange in a stably stratified ocean. Izv. Acad. Sci. USSR, Atmos. Ocean. Phys. 1, 493-497.

Park, Y. G., Whitehead, J. A. \& Gnanadeskian, A. 1994 Turbulent mixing in stratified fluids - layer formation and energetics. J. Fluid Mech. 279, 279-311.

Podesta, J. J. 2008 Laws for third-order moments in homogeneous anisotropic incompressible magnetohydrodynamic turbulence. J. Fluid Mech. 609, 171-194.

Politano, H. \& Pouquet, A. 1998 Von Karman-Howarth equation for MHD and its consequences on third-order longitudinal structure and correlation functions. Phys. Rev. E 57, R21-R24.

Praud, O., Fincham, A. M. \& Sommeria, J. 2005 Decaying grid turbulence in a strongly stratified fluid. J. Fluid Mech. 522, 1-33.

RILEY, J. J. \& DE BRUYN KOPS, S. M. 2003 Dynamics of turbulence strongly influenced by buoyancy. Phys. Fluids 15 (7), 2047-2059.

Riley, J. J. \& LELONG, M.-P. 2000 Fluid motions in the presence of strong stable stratification. Annu. Rev. Fluid Mech. 32, 613-657.

Riley, J. J. \& LindBORG, E. 2008 Stratified turbulence: a possible interpretation of some geophysical turbulence measurements. J. Atmos. Sci. 65, 2416-2424.

Staquet, C. \& Sommeria, J. 2002 Internal gravity waves: from instabilities to turbulence. Annu. Rev. Fluid Mech. 34, 559-593.

Waite, M. L. 2011 Stratified turbulence at the buoyancy scale. Phys. Fluids 23 (6), 066602.

WAite, M. L. \& BARTEllo, P. 2004 Stratified turbulence dominated by vortical motion. J. Fluid Mech. 517, 281-308. 